\title{
A Programmable Beam Shaping System for Tailoring the Profile of High Fluence Laser Beams
}

J. Heebner, M. Borden, P. Miller, C. Stolz, T. Suratwala, P. Wegner, M. Hermann, M. Henesian, C. Haynam, S. Hunter, K. Christensen, N. Wong, L. Seppala, G. Brunton, E. Tse, A. Awwal, M. Franks, E. Marley, K. Williams, M. Scanlan, T. Budge, M. Monticelli, D. Walmer, S. Dixit, C. Widmayer, J. Wolfe, J. Bude, K. McCarty, J. DiNicola

November 22, 2010

2010 Boulder Conference Boulder, CO, United States September 26, 2010 through September 29, 2010 
This document was prepared as an account of work sponsored by an agency of the United States government. Neither the United States government nor Lawrence Livermore National Security, LLC, nor any of their employees makes any warranty, expressed or implied, or assumes any legal liability or responsibility for the accuracy, completeness, or usefulness of any information, apparatus, product, or process disclosed, or represents that its use would not infringe privately owned rights. Reference herein to any specific commercial product, process, or service by trade name, trademark, manufacturer, or otherwise does not necessarily constitute or imply its endorsement, recommendation, or favoring by the United States government or Lawrence Livermore National Security, LLC. The views and opinions of authors expressed herein do not necessarily state or reflect those of the United States government or Lawrence Livermore National Security, LLC, and shall not be used for advertising or product endorsement purposes. 


\title{
A Programmable Beam Shaping System for Tailoring the Profile of High Fluence Laser Beams
}

\author{
John Heebner, Michael Borden, Phil Miller, Christopher Stolz, Tayyab Suratwala, Paul Wegner, \\ Mark Hermann, Mark Henesian, Chris Haynam, Steve Hunter, Kim Christensen, Nan Wong, \\ Lynn Seppala, Gordon Brunton, Eddy Tse, Abdul Awwal, Mark Franks, Ed Marley, Kevin \\ Williams, Michael Scanlan, Tracy Budge, Marcus Monticelli, Dan Walmer, Sham Dixit, Clay \\ Widmayer, Justin Wolfe, Jeff Bude, Kelly McCarty, and Jean-Michel DiNicola
}

Lawrence Livermore National Laboratory, 7000 East Avenue, Livermore, CA 94551

\begin{abstract}
Customized spatial light modulators have been designed and fabricated for use as precision beam shaping devices in fusion class laser systems. By inserting this device in a low-fluence relay plane upstream of the amplifier chain, "blocker" obscurations can be programmed into the beam profile to shadow small isolated flaws on downstream optical components that might otherwise limit the system operating energy. In this two stage system, $1920 \times 1080$ bitmap images are first imprinted on incoherent, $470 \mathrm{~nm}$ address beams via pixilated liquid crystal on silicon (LCoS) modulators. To realize defined masking functions with smooth apodized shapes and no pixelization artifacts, address beam images are projected onto custom fabricated optically-addressable light valves. Each valve consists of a large, single pixel liquid cell in series with a photoconductive Bismuth silicon Oxide (BSO) crystal. The BSO crystal enables bright and dark regions of the address image to locally control the voltage supplied to the liquid crystal layer which in turn modulates the amplitude of the coherent beams at $1053 \mathrm{~nm}$. Valves as large as $24 \mathrm{~mm}$ x $36 \mathrm{~mm}$ have been fabricated with low wavefront distortion $(<0.5$ waves) and antireflection coatings for high transmission ( $>90 \%)$ and etalon suppression to avoid spectral and temporal ripple. This device in combination with a flaw inspection system and optic registration strategy represents a new approach for extending the operational lifetime of high fluence laser optics.
\end{abstract}

Keywords: spatial light modulators, liquid crystal, bismuth silicon oxide, beam shaping, optical damage, high fluence, HEDP

\section{INTRODUCTION}

Megajoule class lasers for inertial confinement fusion require large-aperture optics capable of withstanding high fluence operation at ultraviolet (UV) wavelengths. ${ }^{1}$ In the past decade, work associated with the construction and commissioning of the National Ignition Facility has led to major advances in the manufacturing and processing of high damage threshold UV optics. ${ }^{2}$ While the number of damage-relevant flaws on these optics is very low, it is not yet zero, and the few flaws that remain can initiate damage with the potential to grow in size on subsequent high fluence laser shots. The ability to temporarily shadow, or "block" these few isolated sites from the high fluence laser pulse enhances operational flexibility by enabling uninterrupted use of the facility at near peak laser performance until such time as the optic can be removed, repaired offline, and replaced.

The obscurations that produce the shadows, referred to as "blockers" are introduced in the low fluence, infrared region of the laser upstream of the main amplifier chain. By imaging and aligning the shadows of the blockers onto flaws in the output UV optics, we can ensure that the flaws are protected at the expense of a modest loss of beam area. Initially, we introduced static chrome masks each containing a single "blocker" obscuration into the Pre Amplifier Modules (PAMs) feeding the beamlines on NIF. While these solved an immediate need, we preferred a programmable means for introducing this type of blocker in order to provide enhanced flexibility and reliability for NIF operations. Projection display technology has improved the performance and driven down the cost of pixelated incoherent spatial light modulators. Unfortunately, the direct application of these devices to coherent laser beams presents multiple challenges. These include diffraction artifacts resulting from the pixelated matrix, poor throughput, wavefront distortions, and 
spectral distortions resulting from multi-layer reflections converting imposed frequency modulation into undesirable temporal modulation (FM-AM conversion).

A survey of commercial spatial light modulators did not reveal a viable solution that met all the requirements for this beam shaping system. Moreover, there was a strong desire to engineer a transmissive device that would enable a straightforward drop-in retrofit to an existing system of 48 PAMs. Most commercial spatial light modulators are reflective owing to pixel addressing on a silicon backplane and would have required additional complexity (new beampath routing and relay telescopes) to add an extra reflective relay image plane. The project thus adopted transmissive Optically Addressable Light Valve (OALV) technology. ${ }^{3,4}$ This method leverages incoherent, pixelated display technology to define incoherent images, but eliminates the artifacts associated with modulating coherent beams through use of a custom, single-pixel analog modulator.

\section{OPTICALLY ADDRESSABLE LIGHT VALVE (OALV) DEVICE DESCRIPTION}

The beam shaping hardware consists of two stages. In the first stage, a bitmapped image is written onto an auxiliary incoherent address beam derived from a $470 \mathrm{~nm}$ LED source. The image can be written using any of a number of commercially available projection display technologies such as a standard 1920 x 1080 pixel Liquid Crystal on Silicon (LCoS) modulator as found on rear projection television displays. The address beam carrying the image is then projected upon the OALV which consists of a large, single pixel twisted nematic liquid crystal cell in series with a layer of photoconductive Bismuth Silicon Oxide (BSO). While the single pixel is biased with a common voltage applied to Indium Tin Oxide (ITO) coatings across its entire aperture, the photoconductive layer enables the intensity of the (above bandgap) address beam to locally control the voltage supplied to the liquid crystal layer which in turn modulates the polarization of the coherent beam at $1053 \mathrm{~nm}$. A downstream polarizer enables the polarization modulation to be manifested as an amplitude modulation. As a result, this second stage imprints the incoherent pattern onto the coherent laser beam in a manner that naturally yields smooth apodized patterns free from spurious pixelization artifacts. Figure 1 depicts the OALV architecture and explains the device operation when addressed with a bitmapped image.

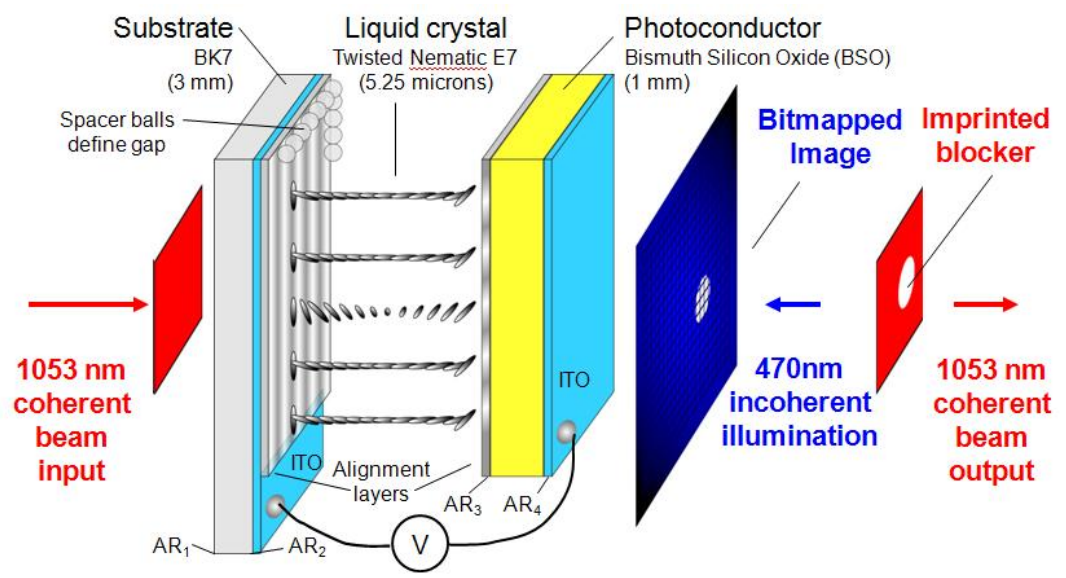

Figure 1. Architecture and operation of the OALV. The OALV is on where blue light is on, "shorting out" the BSO layer and thus permitting a fraction of the applied voltage to appear across the liquid crystal layer. Blockers are imprinted where blue light is absent. A polarizer (not shown in the schematic) downstream of the device enables the polarization rotation to be manifested as an amplitude modulation.

Valves as large as $24 \mathrm{~mm} \times 36 \mathrm{~mm}$ were custom designed and fabricated with low wavefront distortion and antireflection coatings enabling both high transmission and etalon suppression to avoid spectral and temporal ripple. Figure 2 displays an OALV used on NIF. Figure 3 displays a photograph of one of the 48 PAMs upgraded with a Programmable Spatial Shaper package, a photograph of an installed Optically Addressable Light Valve, and a $1053 \mathrm{~nm}$ beam profile imprinted with an array of 9 blockers. 


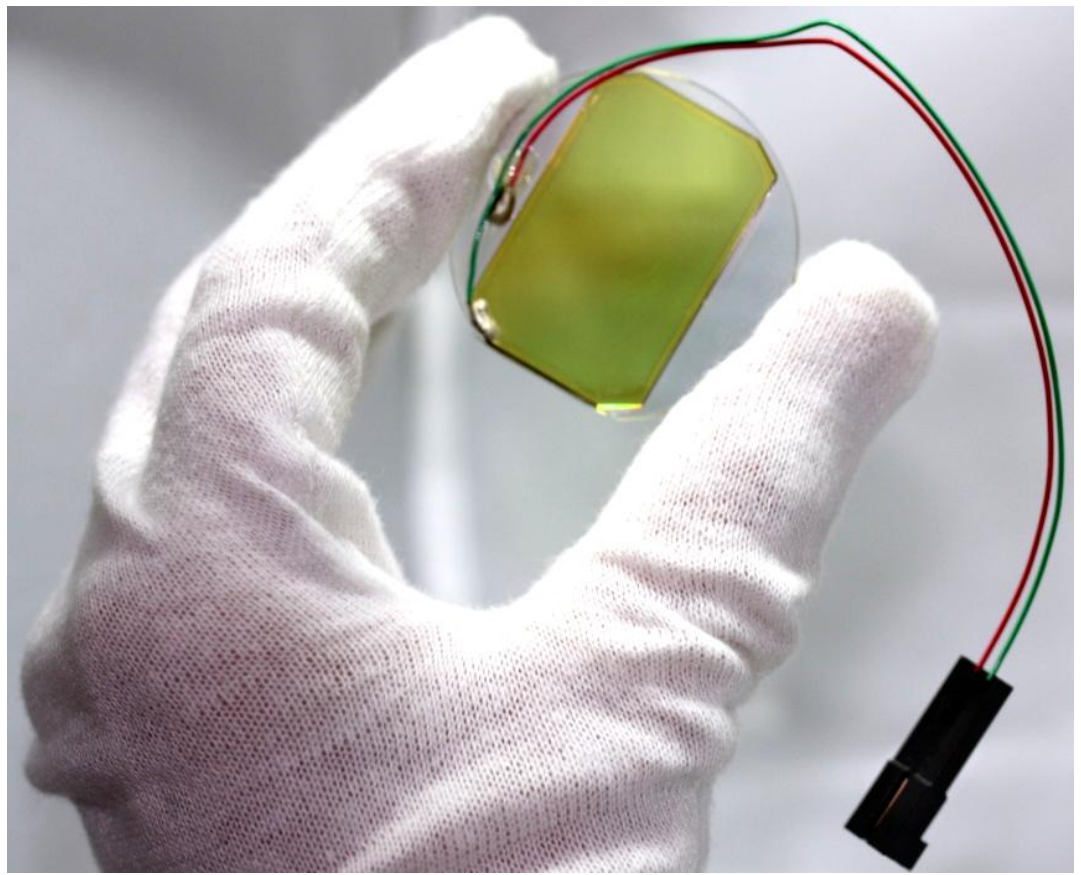

Figure 2. Optically Addressable Light Valve (OALV) custom engineered for shaping the beam profile at the frontend of the laser system at the National Ignition Facility. The valve imprints an incoherent image onto the coherent laser beams without introducing spurious artifacts.

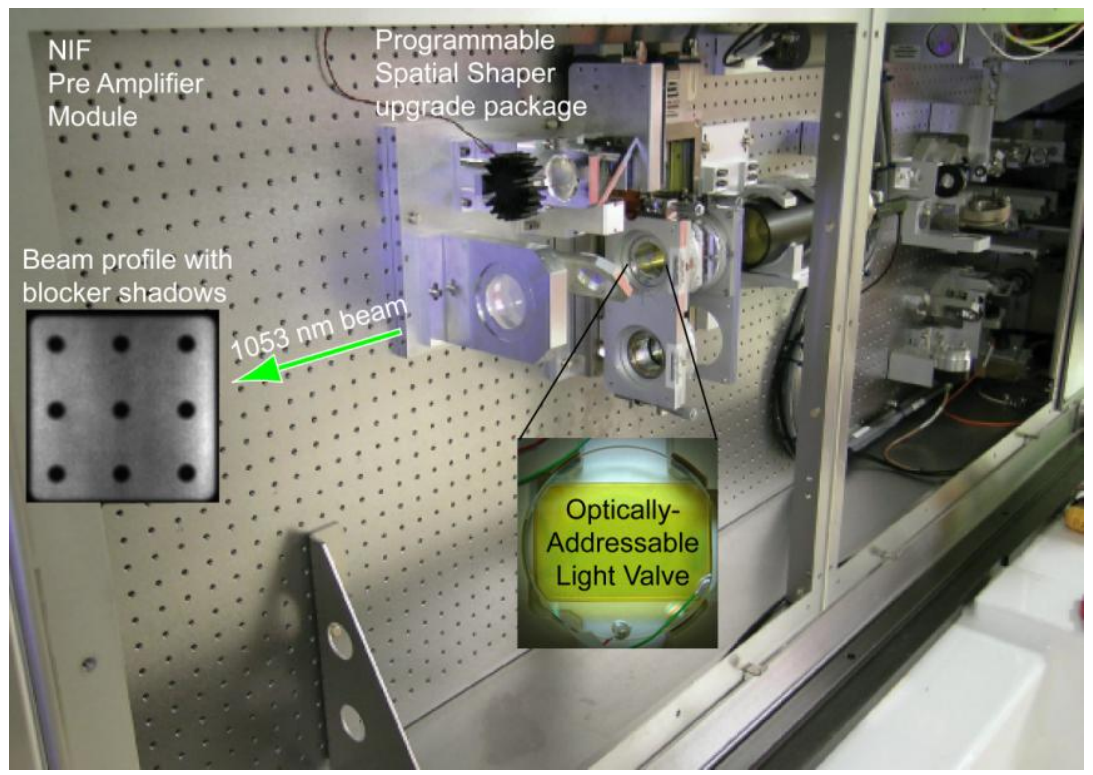

Figure 3. One of 48 PAMs containing an upgrade package to deliver programmable spatial-shaping capability to the National Ignition Facility (NIF)'s beamlines. The packages each contain an optically addressable liquid-crystal light valve that imprints an incoherent image onto the coherent laser beams without introducing spurious artifacts. 


\section{SYSTEM PERFORMANCE}

The clear aperture of the OALV accommodates an $18 \mathrm{~mm} \times 18 \mathrm{~mm}$ square beam profile. At its installed, upstream location, the beam fluence is less than $5 \mathrm{~mJ} / \mathrm{cm}^{2}$. Downstream, the beam is magnified and further amplified until it is 372 $\mathrm{mm} \times 372 \mathrm{~mm}$ operating at $1 \omega(1053 \mathrm{~nm})$ fluences up to $16 \mathrm{~J} / \mathrm{cm}^{2}$ and $3 \omega(351 \mathrm{~nm})$ fluences up to $8 \mathrm{~J} / \mathrm{cm}^{2}$. Using this technique, we are able to realize arbitrarily defined masks with smooth shapes, high transmission (>90\%), low wavefront distortion $(<0.5$ waves), and without spectral distortions. Blocker diameters were carefully chosen as a tradeoff between maintaining usable beam area and minimizing diffraction ripples at edges that can intensify and lead to damage. Propagation studies suggest that the blocker holes can be as small as $1 \mathrm{~mm}$ at the OALV $(2 \mathrm{~cm}$ on the magnified beam downstream) before edge diffraction coupled with Kerr nonlinear effects introduce significant modulation on the beam. ${ }^{5}$ Figure 4 displays line-outs on linear and logarithmic scales through a typical blocker profile demonstrating that a programmable means of introducing our desired blocker shape has been achieved.
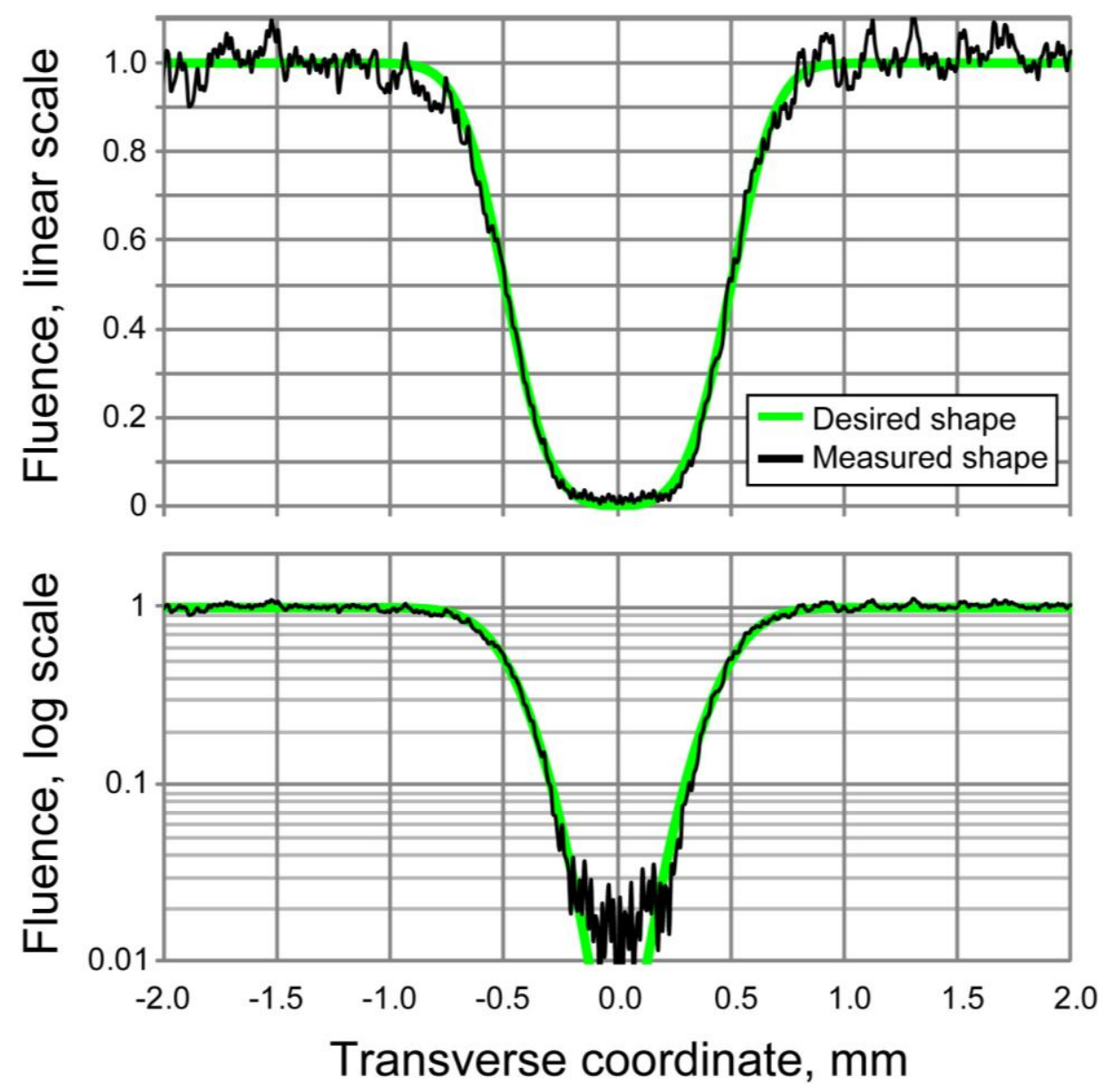

Figure 4. Through-center lineout of a blocker obscuration (on linear and logarithmic scales), demonstrating that we can meet the desired shape requirement. 


\section{CONCLUSION}

The new generation of high energy laser systems for fusion research will rely on continuing advances in high damage resistant optics. Active equipment protection in the form of programmable beam shaping will nevertheless offer significant advantages for flexible and reliable operations. A retrofit of each of the 48 NIF PAMs with this new upgrade package was completed in February, 2010. These devices in combination with a flaw inspection system and optic registration strategy represent a new approach for extending the operational lifetime of high fluence laser optics. Future work will involve optimizing the blocker shapes, and adapting the system to extract the most energy while operating the system safely and reliably.

\section{ACKNOWLEDGEMENTS}

We thank CEA/Cesta and Meadowlark Optics Inc. for contributions to this effort. This work was performed under the auspices of the U.S. Department of Energy by Lawrence Livermore National Laboratory under Contract DE-AC5207NA27344.

\section{REFERENCES}

[1] Lindl, J. D., Amendt, P., Berger, R. L., Glendinning, S. G., Glenzer, S. H., Haan, S. W., Kauffman, R. L.,Landen, O. L., and Suter, L. J., "The physics basis for ignition using indirect-drive targets on the National Ignition Facility," Physics of Plasmas 11, 339-491 (2004).

[2] Suratwala, T., "Optical fabrication and post processing techniques for improving laser damage resistance of fused silica optics," Optical Fabrication and Testing, Paper OWA1 (2010).

[3] Bleha, et al., Opt. Eng. 17, 371 (1978).

[4] Aubourg, P., Huignard, J. P., Hareng, M., and Mullen, R. A., "Liquid crystal light valve using bulk monocrystalline $\mathrm{Bi}_{12} \mathrm{SiO}_{20}$ as the photoconductive material," Applied Optics 21, 3706-3712 (October 1982).

[5] Bahk, S.-W., Zuegel, J. D., Fienup, J. R., and Widmayer, C. C., "Spot-shadowing optimization to mitigate damage growth in a high-energy-laser amplifier chain," Applied Optics 47, 6586-6593 (2008). 